\title{
Article \\ High-Resolution Separation of Nanoparticles Using a Negative Magnetophoretic Microfluidic System
}

\author{
Lin Zeng ${ }^{1}$, Xi Chen ${ }^{1}$, Rongrong Zhang ${ }^{1}$, Shi Hu ${ }^{1}$, Hongpeng Zhang ${ }^{2}$, Yi Zhang ${ }^{3}$ and Hui Yang ${ }^{1, *}$ \\ 1 Laboratory of Biomedical Microsystems and Nano Devices, Bionic Sensing and Intelligence Center, \\ Institute of Biomedical and Health Engineering, Shenzhen Institute of Advanced Technology, \\ Chinese Academy of Sciences, Shenzhen 518055, China; lin.zeng@siat.ac.cn (L.Z.); xi.chen@siat.ac.cn (X.C.); \\ zhangrr@siat.ac.cn (R.Z.); shi.hu@siat.ac.cn (S.H.) \\ 2 Marine Engineering College, Dalian Maritime University, Dalian 116026, China; zhppeter@dlmu.edu.cn \\ 3 Center for Medical AI, Institute of Biomedical and Health Engineering, Shenzhen Institute of Advanced \\ Technology, Chinese Academy of Sciences, Shenzhen 518055, China; yi.zhang3@siat.ac.cn \\ * Correspondence: hui.yang@siat.ac.cn; Tel.: +86-0755-86392675
}

check for updates

Citation: Zeng, L.; Chen, X.; Zhang, R.; Hu, S.; Zhang, H.; Zhang, Y.; Yang, H. High-Resolution Separation of Nanoparticles Using a Negative Magnetophoretic Microfluidic System. Micromachines 2022, 13, 377 https://doi.org/10.3390/mi13030377

Academic Editor: Xiangchun Xuan

Received: 29 December 2021

Accepted: 24 February 2022

Published: 26 February 2022

Publisher's Note: MDPI stays neutral with regard to jurisdictional claims in published maps and institutional affiliations.

Copyright: (c) 2022 by the authors. Licensee MDPI, Basel, Switzerland. This article is an open access article distributed under the terms and conditions of the Creative Commons Attribution (CC BY) license (https:// creativecommons.org/licenses/by/ $4.0 /)$.

\begin{abstract}
The separation and purification of a sample of interest is essential for subsequent detection and analysis procedures, but there is a lack of effective separation methods with which to purify nano-sized particles from the sample media. In this paper, a microfluidic system based on negative magnetophoresis is presented for the high-resolution separation of nanoparticles. The system includes on-chip magnetic pole arrays and permalloys that symmetrically distribute on both sides of the separation channel and four permanent magnets that provide strong magnetic fields. The microfluidic system can separate $200 \mathrm{~nm}$ particles with a high purity from the mixture $(1000 \mathrm{~nm}$ and $200 \mathrm{~nm}$ particles) due to a magnetic field gradient as high as 10,000 T/m being generated inside the separation channel, which can provide a negative magnetophoretic force of up to $10 \mathrm{pN}$ to the $1000 \mathrm{~nm}$ particle. The overall recovery rate of the particles reaches $99 \%$, the recovery rate of $200 \mathrm{~nm}$ particles is $84.2 \%$, and the purity reaches $98.2 \%$. Compared with the existing negative magnetophoretic separation methods, our system not only exhibits high resolution on particle sizes $(800 \mathrm{~nm})$, but also improves the sample processing throughput, which reaches $2.5 \mu \mathrm{L} / \mathrm{min}$. The microfluidic system is expected to provide a new solution for the high-purity separation of nanoparticles, as well as nanobiological samples.
\end{abstract}

Keywords: negative magnetophoresis; nanoparticles; separation; microfluidic chip

\section{Introduction}

Nanobioparticles such as viruses, subcellular organelles, extracellular vesicles, etc., have shown their important roles in disease diagnosis and treatment in recent years, but there is a lack of efficient methods with which to separate and extract nanobioparticles with high purity [1]. Microfluidic devices have become a popular tool for biological sample processing and analysis due to their small size, fast response, low cost, and feasibility to develop into highly integrated portable devices [2]. Since the critical dimension of a microfluidic channel is close to that of micrometer-sized particles, such as cells, a microfluidic device therefore shows advantages that are usually difficult to achieve through traditional technology in the separation and detection of microparticles [3,4]. To date, microfluidic-based separation techniques using magnetic field [5,6], surface acoustic wave [7], dielectrophoresis [8], optics [9], inertia effect [10], and deterministic lateral displacement [11], etc., have become technically mature in cell separation. However, when these techniques are applied to separate nanoparticles, good results can hardly be achieved [12]. Various methods have been proposed to separate and purify nanoparticles $[13,14]$, but some of them would cause damage to biological samples, for example, due to the Joule heat generated by the optical field [15] and the surface potential generated by dielectrophoresis [16]. 
Among various techniques, magnetic separation provides a relatively more straightforward solution that would not bring damage to biological samples, and the magnetic field is flexible and controllable $[17,18]$. Magnetic separation can be categorized into labeled and label-free methods based on the different methods of processing biological samples, corresponding to positive magnetophoresis and negative magnetophoresis, respectively. Positive magnetophoresis requires magnetic beads to label the target biosamples, and therefore the biosamples can be precisely controlled by an external magnetic field, however, in this case, requiring magnetic beads as the solid substrate to capture the biological samples and additional elution protocols $[19,20]$. Negative magnetophoresis has been recently proposed for cell separation and has drawn much attention due to its label-free nature. By using paramagnetic salt solution or ferrofluid as the sample medium, negative magnetophoresis can separate the non-magnetic biosamples based on their size difference [21,22]. However, the size resolution, i.e., the minimum difference of the sizes of the biosamples that can be separated from a mixture state often remains at the micrometer-scale, since permeability of the magnetic solution and magnetic field gradients are usually very low, therefore the magnetic separation force is also small $[23,24]$.

The development of ferrofluid-based negative magnetophoresis started from the use of external permanent magnets to separate microparticles and cells $[25,26]$. In order to improve the size resolution, other forces such as viscoelasticity [27], inertial force [28], and centrifugal force [29] are combined with the negative magnetophoretic force in a single chip. Recently, new magnetic structures have been used to improve the magnetic field gradient, for example, utilizing four permanent magnets presented by Mao et al. [30], as well as generating an on-chip magnetic pole array in our previously published work [31], both of which greatly improve the magnetic field gradient and successfully achieve a separation of $200 \mathrm{~nm}$ and $1000 \mathrm{~nm}$ particles, however the sample throughput in these two works is not high enough.

Here, we present a new microfluidic system for the separation of nanoparticles based on negative magnetophoresis. With the unique design of the microfluidic and magnetic structures in the system, nanometer-sized particles can be efficiently separated with high purity and high sample throughput.

\section{Materials and Methods}

\subsection{Chip Design}

Details on the chip design are shown in Figure 1. The system consisted of four external permanent magnets, two high-permeability alloys (permalloy, 1J85), two on-chip magnetic pole arrays, and a separation channel. The external permanent magnets provide a strong magnetic field, which can be conducted to the on-chip magnetic pole arrays through the high-permeability alloys, then the magnetic pole arrays arranged on both sides of the separation channel can generate a high-gradient and high-intensity magnetic field in the separation channel. The ferrofluid, which is made up of $\mathrm{Fe}_{3} \mathrm{O}_{4}$ nanoparticles $(10 \mathrm{~nm})$ suspended in water, generated a concentration difference in the separation channel; the higher the magnetic field gradient is, the higher the concentration of the $\mathrm{Fe}_{3} \mathrm{O}_{4}$ nanoparticles, therefore the non-magnetic particles suspended in the ferrofluid are subjected to a pressure that is caused by the concentration difference of the $\mathrm{Fe}_{3} \mathrm{O}_{4}$ nanoparticles, which is referred to as the "negative magnetophoretic force", and the direction towards to the low magnetic field gradient area. 


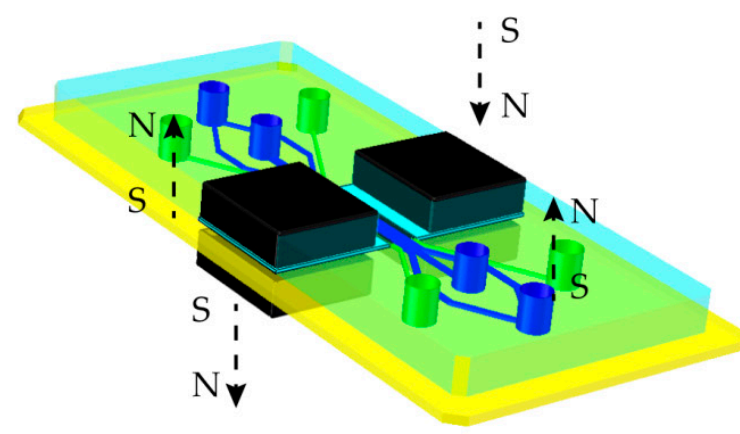

(a)

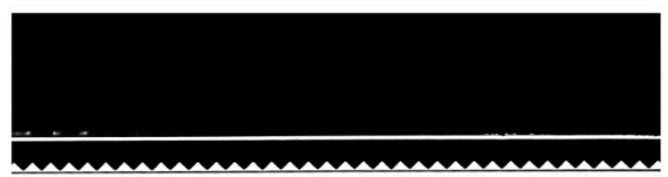

$600 \mu \mathrm{m}$

(c)

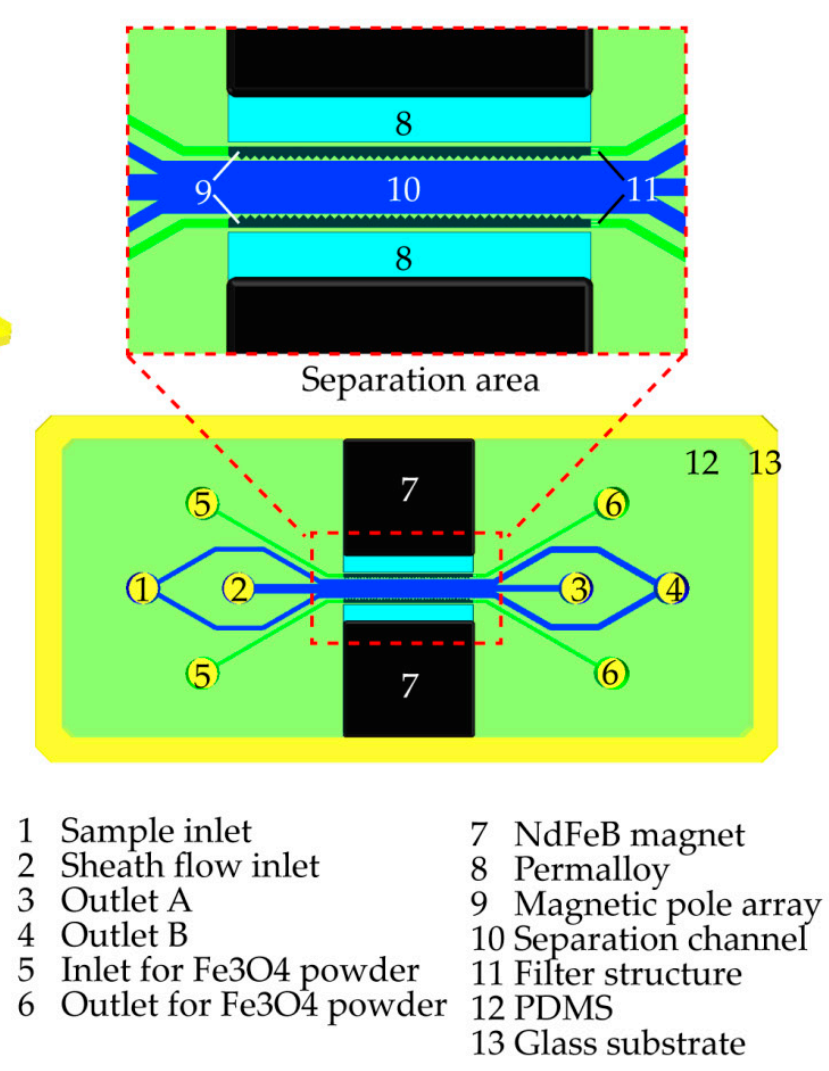

(b)

Figure 1. Design and image of the microfluidic system: (a) Overall structure of the system; (b) Schematic illustration on the system design; (c) Microscopic image on the separation area.

In this chip, the sheath fluid can focus the sample particles on both sides of the separation channel (that is, the area close to the magnetic pole arrays). The $200 \mathrm{~nm}$ particles can flow into the outlet channels on both sides and finally flow into outlet B. The magnetic field gradient and magnetic field strength in the side area are relatively high, which can make the $1000 \mathrm{~nm}$ particles deflect toward the center of the separation channel by a strong negative magnetophoretic force, and the $1000 \mathrm{~nm}$ particles finally flow into outlet A. Thereby, particles with different sizes can be separated and collected from the different outlets of the microfluidic system.

\subsection{Fabrication of the Microfluidic System}

The fabrication of the system was divided into two steps. The first step was to fabricate a conventional microfluidic chip using soft lithography. The height of all microchannels was $50 \mu \mathrm{m}$, the width of the two sample inlet channels was $100 \mu \mathrm{m}$, the width of the sheath flow inlet channel was $1000 \mu \mathrm{m}$, the width of the separation channel was $1200 \mu \mathrm{m}$ (as shown in Figure 1b), and the width of three outlet channels at the end was $400 \mu \mathrm{m}$. The second step was to build a high-gradient magnetic field structure on the chip. The $\mathrm{Fe}_{3} \mathrm{O}_{4}$ powders (5-10 $\mu \mathrm{m}$ in diameter) mixed with ethanol were first injected into the magnetic pole channels to form the magnetic pole arrays. The width of the magnetic pole channels on both sides was $100 \mu \mathrm{m}$, and the distance from the tip of the magnetic pole to the separation channel was $10 \mu \mathrm{m}$. Then, the permalloy (1J85, $30 \mu \mathrm{m}$ in thickness, Dongguan Saijing Special Alloy Co., Ltd., Dongguan, China) was embedded in both sides of the magnetic pole arrays; here, we reserved a permalloy embedded channel $(26 \mathrm{~mm}$ in width and $50 \mu \mathrm{m}$ in height) on the sides of the magnetic pole arrays, so that the rectangular permalloy could be precisely embedded in both sides of the magnetic pole arrays under the microscope. Finally, the magnets (N52, $25.4 \mathrm{~mm} \times 12.7 \mathrm{~mm} \times 3.175 \mathrm{~mm}$ ) were added to the chip through 
a fixed mold. The distance from the permalloy to the magnetic pole channel was $30 \mu \mathrm{m}$, and the length of the magnetic pole array was $26 \mathrm{~mm}$.

\subsection{Experimental Setup}

In order to verify the effect of the separation system, fluorescent polystyrene particles (nonmagnetic) with sizes of $200 \mathrm{~nm}$ (yellow-green, Thermo Fisher Scientific, 35,000 particles/ $\mu \mathrm{L}$ ) and $1000 \mathrm{~nm}$ (glacial blue, Bangs Laboratories, Inc, Fishers, IN, USA., $\sim 3500$ particles/ $\mu \mathrm{L}$ ) were mixed in the diluted ferrofluid (EMG 805, 3.6\%). First, the ratio setting was chosen to mimic the concentration ratio of micro-extracellular vesicles (microEVs, $>800 \mathrm{~nm}$ ) and small-extracellular vesicles (sEVs, $<200 \mathrm{~nm}$ ) in blood plasma [13], since the sEVs are the typical nanobiological samples which may be separated using our system in the future. When the concentration of the $1000 \mathrm{~nm}$ particle increases, the interaction between the two kinds of the particles increases as well. On one hand, it may slightly affect the separation performance; on the other hand, the impact is extremely limited based on the previous reference [24]. Then, the mixture sample and the sheath fluid (diluted ferrofluid without fluorescent particles) were injected into the chip. In the experiments, the sample concentration and flow rate were optimized first. Fluorescence trajectories of the particles were recorded using an inverted microscope (Zeiss Axio Observer 7, Carl Zeiss Microscopy GmbH, Jena, Germany) equipped with appropriate fluorescent filter sets and an sCMOS camera (ORCA-Flash4.0-V3, Hamamatsu, Japan). Then, we used the optimal parameters to separate the fluorescent particles. The separation efficiency and the recovery rate of $200 \mathrm{~nm}$ particles were then analyzed using a flow cytometer (FCM, CytoFLEX S, Beckman Coulter, Atlanta, GA, USA).

\section{Results}

We first analyzed the force acting on the particles in the microfluidic channel, and the magnetic field of the chip and the particle trajectories were simulated using the finite element method in Comsol Multiphysics, and finally the separation experiments were carried out to verify the performance of the system.

\subsection{Theory and Mechanism}

When particles are transported through the microchannel, they flow forward under the hydrodynamic drag force $\boldsymbol{F}_{d}$, and the negative magnetophoretic force $\boldsymbol{F}_{m}$ acts on the particles when they enter the separation area, as shown in Figure 2a. The hydrodynamic drag force $\boldsymbol{F}_{d}$ is described by the following equation [32]:

$$
\boldsymbol{F}_{d}=3 \pi \eta_{f} D_{p} f_{D}\left(\boldsymbol{u}_{f}-\boldsymbol{u}_{p}\right)
$$

where $\eta_{f}$ is the dynamic viscosity of the ferrofluid, $D_{p}$ is the diameter of the particle, $\boldsymbol{u}_{f}$ and $\boldsymbol{u}_{p}$ are velocity vectors of ferrofluid and particle, respectively. $f_{D}$ is the hydrodynamic drag force coefficient that is determined by the structure of microchannel and the particle position:

$$
f_{\mathrm{D}}=\left[1-\frac{9}{16}\left(\frac{D_{\mathrm{p}}}{D_{\mathrm{p}}+2 L}\right)+\frac{1}{8}\left(\frac{D_{\mathrm{p}}}{D_{\mathrm{p}}+2 L}\right)^{3}-\frac{45}{256}\left(\frac{D_{\mathrm{p}}}{D_{\mathrm{p}}+2 L}\right)^{4}-\frac{1}{16}\left(\frac{D_{\mathrm{p}}}{D_{\mathrm{p}}+2 L}\right)^{5}\right]^{-1}
$$

where $L$ is shortest distance between the surface of the particle and the channel wall. 


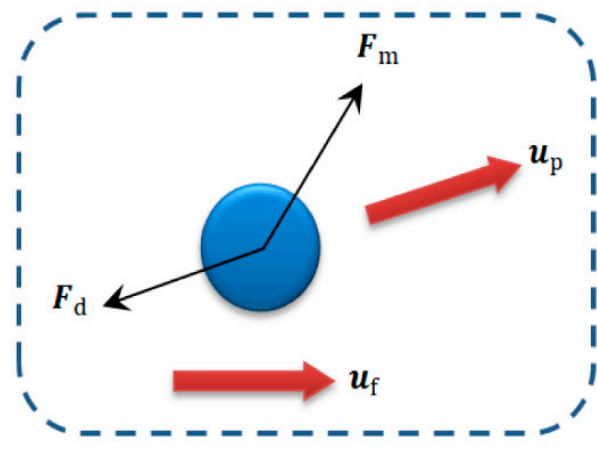

(a)

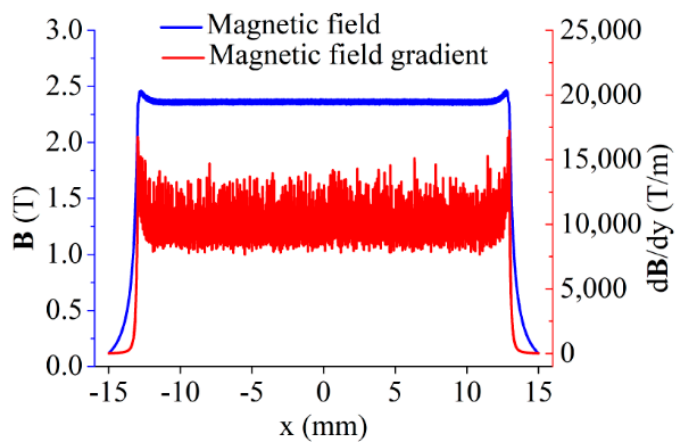

(c)

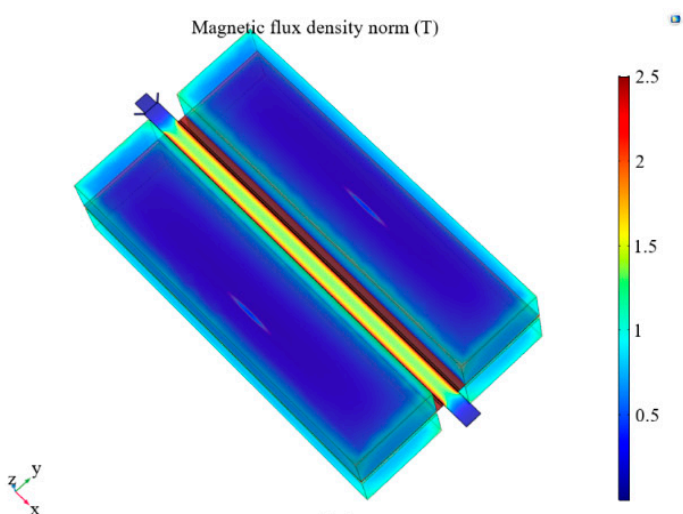

(b)

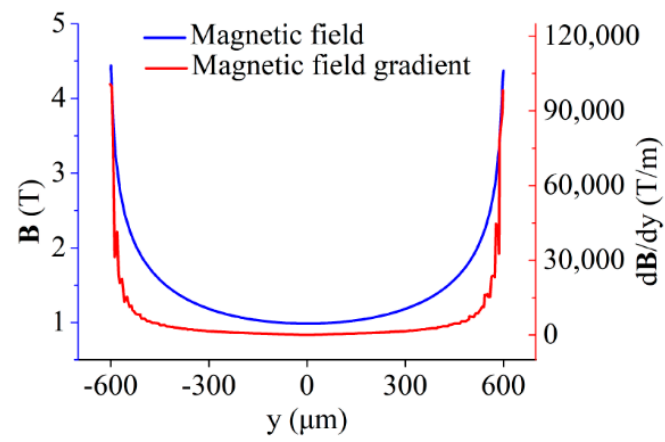

(d)

Figure 2. Simulation results of the magnetic field and particle trajectories: (a) The force analysis of the particle in separation channel; (b) The magnetic field distribution of separation area; (c) The magnetic field and its gradient in separation area along $x$-axis; (d) The magnetic field and its gradient in separation area along $y$-axis.

The negative magnetophoretic force $\boldsymbol{F}_{m}$ is described by the following equation [33]:

$$
\boldsymbol{F}_{m}=\frac{V_{p}\left(\chi_{p}-\chi_{f}\right)}{\mu_{0}}(\boldsymbol{B} \cdot \nabla) \boldsymbol{B}
$$

where $V_{p}$ is the volume of the particle, $\mu_{0}$ is the permeability of free space, $\boldsymbol{B}$ is the magnetic induction, $\chi_{p}$ and $\chi_{f}$ are the magnetic susceptibilities of particle and ferrofluid, respectively. The negative magnetophoretic force $\boldsymbol{F}_{m}$ is proportional to the particle volume.

The particles of different sizes eventually enter different outlets following different trajectories that are determined by these two forces. The motion control equation is:

$$
m_{\mathrm{p}} \frac{\mathrm{d} \boldsymbol{u}_{\mathrm{p}}}{\mathrm{d} t}=\boldsymbol{F}_{\mathrm{m}}+\boldsymbol{F}_{\mathrm{d}}
$$

Based on the Equations (1)-(4), the particle trajectories can be calculated by the simulation software. The initial velocity $\boldsymbol{u}_{f}$ is set according to the experimental setup.

\subsection{Simulation}

Based on the chip design, a model of the separation area is established in Comsol Multiphysics. In simulation, the "Magnetic fields, No Current" module was used to solve the magnetic field and was applied to all domains; the "Laminar Flow" module was used to solve the flow field and was applied to the separation channel; and the "Particle Tracing for Fluid Flow" module was used to solve the particle-moving trajectories in ferrofluid and was also applied to the separation channel. The parameters used in the simulation are shown in Table 1 [34]. Here, the wall of the separation channel was set to no slip. The free 
tetrahedral mesh of all domains was predefined as finer. While in the separation channel domain, the mesh is refined to extremely fine, the maximum element size is set to $35 \mu \mathrm{m}$, and the minimum element size is set to $3 \mu \mathrm{m}$. The simulation result of the magnetic field is shown in Figure $2 b$. In the separation channel, we analyzed the magnetic field and its gradient along $x$-axis $(y=50 \mu \mathrm{m}$ and $\mathrm{z}=0 \mu \mathrm{m})$ and $\mathrm{y}$-axis $(\mathrm{y}=50 \mu \mathrm{m}$ and $\mathrm{z}=0 \mu \mathrm{m})$; as shown in Figure 2c,d, the origin of the coordinates is at the center of the separation channel. The simulation results show that the magnetic pole arrays in both sides can generate a magnetic field exceeding 2 Tesla (T) in the separation channel, and the highest magnetic field gradient reaches $10,000 \mathrm{~T} / \mathrm{m}$.

Table 1. The parameters used in the simulations.

\begin{tabular}{cc}
\hline Parameters & Value \\
\hline Remanent flus density of the magnets & $1.48 \mathrm{~T}$ \\
Relative permeability of ferrofluid after dilution & 1.00069 \\
Relative permeability of $\mathrm{Fe}_{3} \mathrm{O}_{4}$ powder & 4 \\
Relative permeability of permalloy & 80,000 \\
\hline
\end{tabular}

We calculated the negative magnetophoretic force on $1000 \mathrm{~nm}$ particles in the separation area based on the magnetic field simulation results and Equation (1), as shown in Figure $3 a, b$. When they flow through the separation channel along the channel side wall, the force of $1000 \mathrm{~nm}$ particles can reach $10 \mathrm{pN}$, which is strong enough to separate them from the mixture state.

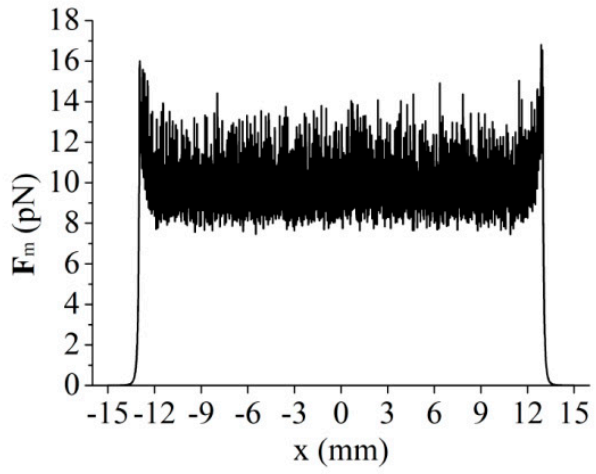

(a)

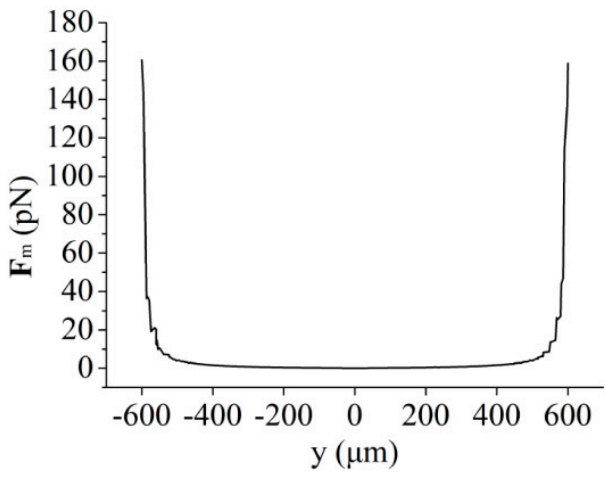

(b)

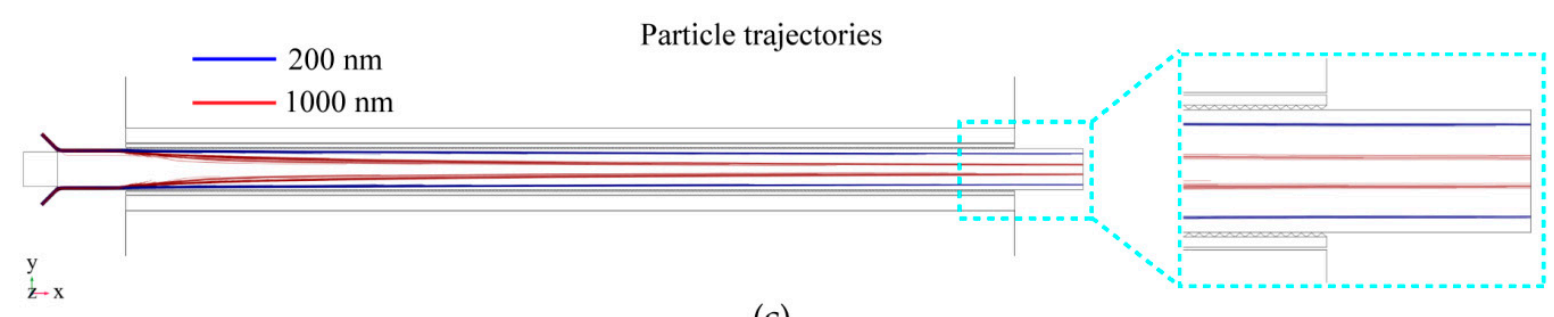

(c)

Figure 3. Calculated negative magnetophoretic force and particle trajectories based on the simulation results: (a) Negative magnetophoretic force acting on $1000 \mathrm{~nm}$ particles in the separation area along $x$-axis and (b) along $y$-axis; (c) The trajectories of $200 \mathrm{~nm}$ and $1000 \mathrm{~nm}$ particles in the simulation.

Based on the magnetic field simulation and negative magnetophoretic force calculation, we further simulated the trajectories of $200 \mathrm{~nm}$ and $1000 \mathrm{~nm}$ particles $(10$ particles/s for each size) in the separation area, as shown in Figure 3c. The negative magnetophoretic force is used to drive the $1000 \mathrm{~nm}$ particles, pushing them further away from both sides of the separation channel and eventually moving into outlet $A$. The results show that the negative magnetophoresis plays a key role in separating the $1000 \mathrm{~nm}$ particles. Since the negative 
magnetophoretic force is proportional to the particle volume, the deflection distance of the $200 \mathrm{~nm}$ particles is therefore very small, and most of the $200 \mathrm{~nm}$ particles can be collected from outlet B. In order to achieve a high recovery rate of $200 \mathrm{~nm}$ particles, the dividing line of the two kinds of particles should be set close to the trajectories of $1000 \mathrm{~nm}$ particles (here, the width of three outlet channels at the end is $400 \mu \mathrm{m})$.

\subsection{Separation Experiments}

In the experiments, the optimal concentration of the ferrofluid was chosen as $0.003 \times(0.01 \%)$, based on our precious study [31]. Here, we first optimized the flow rate of the sample, the results of which are shown in Figure 4. Compared with the simulation results, the trajectories in the experiments are more divergent. For both the $200 \mathrm{~nm}$ and $1000 \mathrm{~nm}$ particles, the trajectories are moving to the side channel as the flow rate increases. In order to optimize the flow rates, we calculated the position distribution of particle trajectories along the $y$-axis direction, which is defined here as dy.

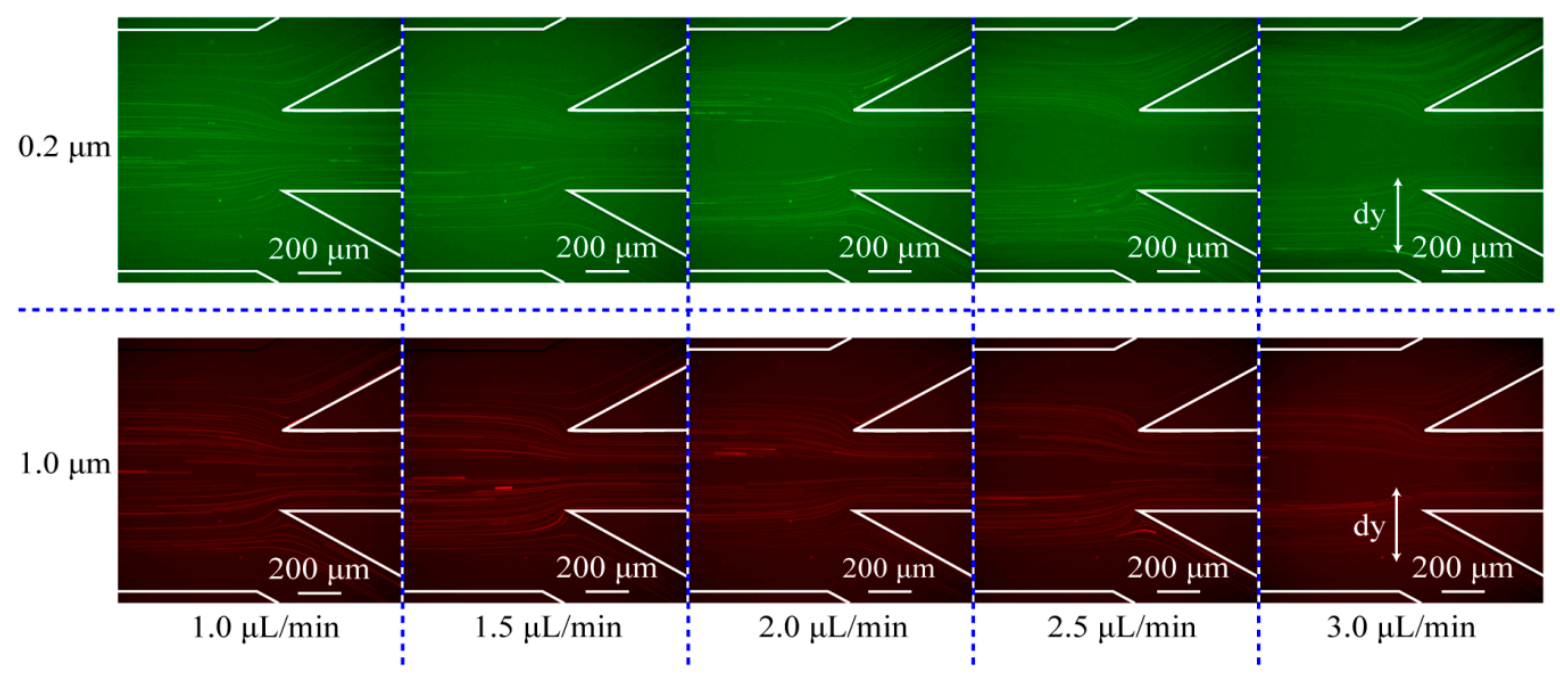

Figure 4. The trajectories of $200 \mathrm{~nm}$ and $1000 \mathrm{~nm}$ particles at different flow rates in the experiments.

The comparison between simulation results and experimental results on the position distribution of particle trajectories at the outlet area under different flow rates of the sample flow are shown in Figure 5. Based on the position distribution, we calculated the widths of the overlap area of the two kinds of particles at different flow rates, and the overlap area demonstrates that the two kinds of particles are not separated effectively. The results show that the overlap width is the shortest at a flow rate of $2.5 \mu \mathrm{L} / \mathrm{min}$, therefore we can conclude that the optimal sample flow rate is $2.5 \mu \mathrm{L} / \mathrm{min}$ (the ratio of the flow rates of the sample flow and the sheath flow is kept as 1:5, the ratio set can ensure that the particles are focused close to the channel wall without dispersing to outlet A when there are no magnets). Under the optimal conditions, most of the $200 \mathrm{~nm}$ particles flow into side outlets and finally converge on outlet B. On the contrary, most of the $1000 \mathrm{~nm}$ particles flow into outlet A through the center channel (Figure 4).

It is noted that the simulation is performed in an ideal condition (the interactions between particles are ignored) and that the trajectories in the simulation are more concentrated than those in the experiments.

In order to quantitatively analyze the particles collected from the two outlets, we used a flow cytometer to count the particles, the results of which are shown in Figure 6. Here, we used FITC and DAPI as the fluorescent detection channels for the $200 \mathrm{~nm}$ and $1000 \mathrm{~nm}$ particles, respectively, and the standard particles of both sizes were gathered in a cluster. The $1000 \mathrm{~nm}$ particles were detected in the upper left corner (red cluster), while the $200 \mathrm{~nm}$ particles were gathered in the lower right corner (green cluster). The overall recovery rate of all particles is $99.0 \%$ (the ratio of the number of particles from outlets to that from the inlet). 
The purity of $200 \mathrm{~nm}$ particles reached $98.2 \%$ (the ratio of the number of $200 \mathrm{~nm}$ particles to all the particles from outlet $\mathrm{B}$ ) and recovery rate was $84.2 \%$ (the ratio of the number of $200 \mathrm{~nm}$ particles from outlet $B$ to that from all outlets), and the resolution reached $800 \mathrm{~nm}$. Using the optimal parameters, the chip can effectively separate $200 \mathrm{~nm}$ particles from the mixed particles of two sizes. Both the theoretical and experimental results prove the high performance of the proposed system.

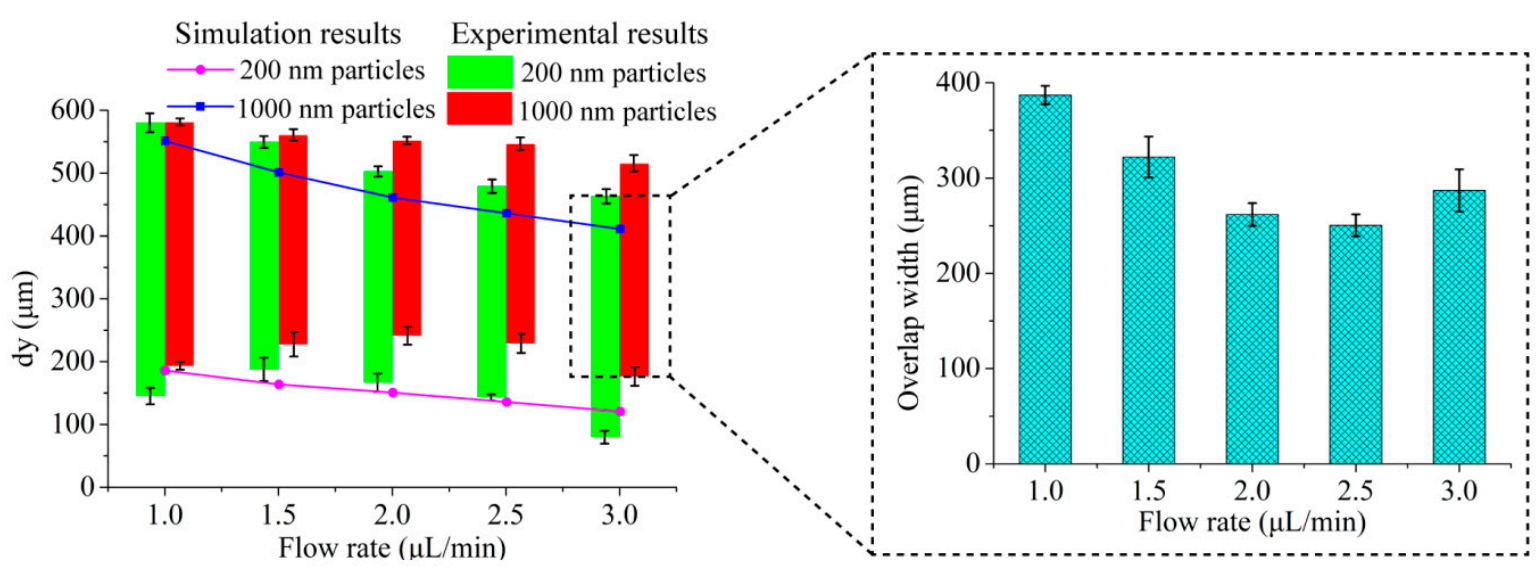

Figure 5. Comparison between simulation results and experimental results on particle distribution along $y$-axis at the outlet area under different flow rates of the sample flow.

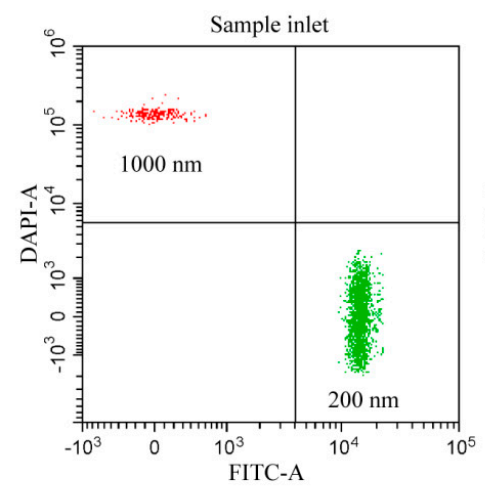

(a)

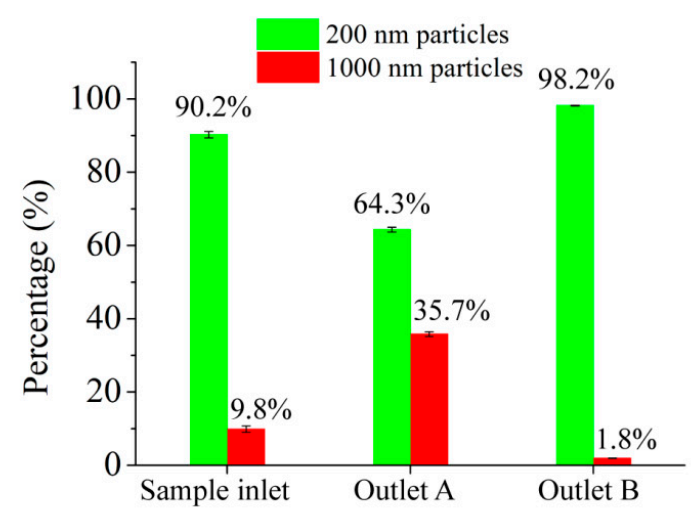

(d)

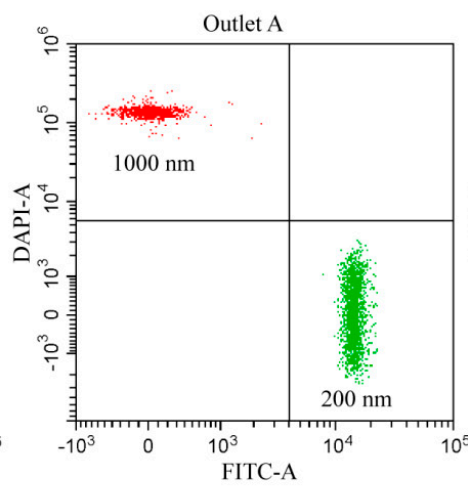

(b)

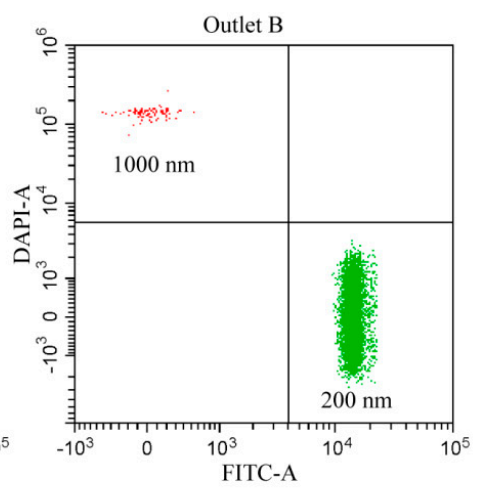

(c)

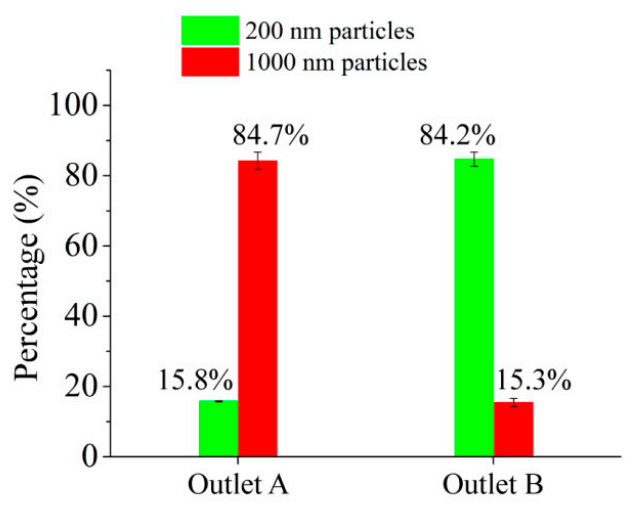

(e)

Figure 6. Flow cytometry test results obtained from: (a) Sample inlet; (b) Outlet A; (c) Outlet B; (d) The proportion of the two kinds of particles at inlet and outlets; (e) The number distribution of the two kinds of particles at both outlets. 


\section{Conclusions}

In this paper, a microfluidic system for the high-resolution nanoparticle separation based on negative magnetophoresis is presented. By combining external magnets, permalloys, and on-chip magnetic pole arrays, the system can generate a magnetic field gradient as high as 10,000 $\mathrm{T} / \mathrm{m}$ inside the separation microchannel, providing a negative magnetophoretic force $(10 \mathrm{pN})$ that is large enough to separate $200 \mathrm{~nm}$ from the mixture samples $(1000 \mathrm{~nm}$ and $200 \mathrm{~nm})$. The recovery rate of $200 \mathrm{~nm}$ particles is $84.2 \%$ and the purity reaches $98.2 \%$. The size resolution of our system reaches $800 \mathrm{~nm}$ and the throughput is improved to $2.5 \mu \mathrm{L} / \mathrm{min}$. We believe that, in future, it may develop into a versatile tool to separate nanometric objects of environmental or biological importance, such as nanoparticles, viruses, or other biological agents.

Author Contributions: Conceptualization, H.Y. and L.Z.; methodology, L.Z.; software, L.Z.; validation, L.Z., X.C. and R.Z.; formal analysis, S.H.; investigation, H.Z.; resources, Y.Z.; data curation, L.Z.; writing—original draft preparation, L.Z.; writing—-review and editing, H.Y.; visualization, H.Y.; supervision, H.Y.; project administration, H.Y.; funding acquisition, H.Y. and L.Z. All authors have read and agreed to the published version of the manuscript.

Funding: This research was funded by the Key-Area Research and Development Program of Guangdong Province (2019B020226004), the National Natural Science Foundation of China (62074155), the Basic and Applied Basic Research Foundation of Guangdong Province (2020A1515110938, 2020A1515110142), SIAT Innovation Program for Excellent Young Researchers (E1G049), Shenzhen Science and Technology Innovation Commission (KCXFZ202002011008124).

Institutional Review Board Statement: Not applicable.

Data Availability Statement: The data that support the findings of this study are available from the corresponding author upon reasonable request.

Acknowledgments: We would like to thank all colleagues and students in our laboratory for helpful discussions.

Conflicts of Interest: The authors declare no conflict of interest.

\section{References}

1. Jeppesen, D.K.; Fenix, A.M.; Franklin, J.L.; Higginbotham, J.N.; Zhang, Q.; Zimmerman, L.J.; Liebler, D.C.; Ping, J.; Liu, Q.; Evans, R.; et al. Reassessment of exosome composition. Cell 2019, 177, 428-445. [CrossRef] [PubMed]

2. Whitesides, G.M. The origins and the future of microfluidics. Nature 2006, 442, 368-373. [CrossRef] [PubMed]

3. Yeo, Y.L.; Chang, H.C.; Chan, P.P.Y.; Friend, J.R. Microfluidic devices for bioapplications. Small 2011, 7, 12-48. [CrossRef] [PubMed]

4. Shen, Y.; Yalikun, Y.; Tanaka, Y. Recent advances in microfluidic cell sorting systems. Sens. Actuators B. 2019, 282, $268-281$. [CrossRef]

5. Gijs, M.A.M.; Lacharme, F.; Lehmann, U. Microfluidic applications of magnetic particles for biological analysis and catalysis. Chem. Rev. 2010, 110, 1518-1563. [CrossRef]

6. Xuan, X. Recent advances in continuous-flow particle manipulations using magnetic fluids. Micromachines 2019, 10, 744. [CrossRef]

7. Li, P.; Mao, Z.; Peng, Z.; Zhou, L.; Chen, Y.; Huang, P.H.; Truica, C.I.; Drabick, J.J.; El-Deiry, W.S.; Dao, M.; et al. Acoustic separation of circulating tumor cells. Proc. Natl. Acad. Sci. 2015, 112, 4970-4975. [CrossRef]

8. Song, H.; Rosano, J.M.; Wang, Y.; Garson, C.J.; Prabhakarpandian, B.; Pant, K.; Klarmann, G.J.; Perantoni, A.; Alvarez, L.M.; Lai, E. Continuous-flow sorting of stem cells and differentiation products based on dielectrophoresis. Lab Chip 2015, 15, 1320-1328. [CrossRef]

9. Qi, X.; Carberry, D.M.; Cai, C.; Hu, S.; Yuan, Z.; Rubinsztein-Dunlop, H.; Guo, J. Optical sorting and cultivation of denitrifying anaerobic methane oxidation archaea. Biomed. Opt. Express 2017, 8, 934-942. [CrossRef]

10. Wu, Z.; Chen, Y.; Wang, M.; Chung, A.J. Continuous inertial microparticle and blood cell separation in straight channels with local microstructures. Lab Chip 2016, 16, 532-542. [CrossRef]

11. Au, S.H.; Edd, J.; Stoddard, A.E.; Wong, K.H.K.; Fachin, F.; Maheswaran, S.; Haber, D.A.; Stott, S.L.; Kapur, R.; Toner, M. Microfluidic isolation of circulating tumor cell clusters by size and asymmetry. Sci. Rep. 2017, 7, 1-10. [CrossRef]

12. Salafi, T.; Zeming, K.K.; Zhang, Y. Advancements in microfluidics for nanoparticle separation. Lab Chip 2017, 17, 11-33. [CrossRef] [PubMed]

13. Wu, M.; Ouyang, Y.; Wang, Z.; Zhang, R.; Huang, P.; Chen, C.; Li, H.; Li, P.; Quinn, D.; Dao, M.; et al. Isolation of exosomes from whole blood by integrating acoustics and microfluidics. Proc. Natl. Acad. Sci. USA 2017, 114, 10584-10589. [CrossRef] [PubMed] 
14. Wunsch, B.H.; Smith, J.T.; Gifford, S.M.; Wang, C.; Brink, M.; Bruce, R.L.; Austin, R.H.; Stolovitzky, G.; Astier, Y. Nanoscale lateral displacement arrays for the separation of exosomes and colloids down to $20 \mathrm{~nm}$. Nat. Nanotechnol. 2016, 11, 936-940. [CrossRef]

15. Kayani, A.A.; Khoshmanesh, K.; Ward, S.A.; Mitchell1, A.; Kalantar-zadeh, K. Optofluidics incorporating actively controlled micro-and nano-particles. Biomicrofluidics 2012, 6, 031501. [CrossRef] [PubMed]

16. Dash, S.; Mohanty, S.; Pradhan, S.; Mishra, B.K. CFD design of a microfluidic device for continuous dielectrophoretic separation of charged gold nanoparticles. J. Taiwan Inst. Chem. Eng. 2016, 58, 39-48. [CrossRef]

17. Xu, H.; Liao, C.; Zuo, P.; Liu, Z.; Ye, B. Magnetic-based microfluidic device for on-chip isolation and detection of tumor-derived exosomes. Anal. Chem. 2018, 90, 13451-13458. [CrossRef]

18. Cao, Q.; Han, X.; Li, L. Configurations and control of magnetic fields for manipulating magnetic particles in microfluidic applications: Magnet systems and manipulation mechanisms. Lab Chip 2014, 14, 2762-2777. [CrossRef]

19. Lee, J.J.; Jeong, K.J.; Hashimoto, M.; Kwon, A.H.; Rwei, A.; Shankarappa, S.A.; Tsui, J.H.; Kohane, D.S. Synthetic ligand-coated magnetic nanoparticles for microfluidic bacterial separation from blood. Nano Lett. 2014, 14, 1-5. [CrossRef]

20. Martin, A.B.; Wu, W.T.; Kameneva, M.V.; Antaki, J.F. Development of a high-throughput magnetic separation device for malaria-infected erythrocytes. Ann. Biomed. Eng. 2017, 45, 2888-2898. [CrossRef]

21. Zhou, Y.; Xuan, X. Diamagnetic particle separation by shape in ferrofluids. Appl. Phys. Lett. 2016, 109, 102405. [CrossRef]

22. Zhao, W.; Cheng, R.; Lim, S.H.; Miller, J.R.; Zhang, W.; Tang, W.; Xie, J.; Mao, L. Biocompatible and label-free separation of cancer cells from cell culture lines from white blood cells in ferrofluids. Lab Chip 2017, 17, 2243-2255. [CrossRef] [PubMed]

23. Kwon, S.; Oh, J.; Lee, M.S.; Um, E.; Jeong, J.; Kang, J.H. Enhanced diamagnetic repulsion of blood cells enables versatile plasma separation for biomarker analysis in blood. Small 2021, 17, 2100797. [CrossRef]

24. Zhao, W.; Zhu, T.; Cheng, R.; Liu, Y.; He, J.; Qiu, H.; Wang, L.; Nagy, T.; Querec, T.D.; Unger, E.R.; et al. Label-free and continuous-flow ferrohydrodynamic separation of HeLa cells and blood cells in biocompatible ferrofluids. Adv. Funct. Mater. 2016, 26, 3990-3998. [CrossRef] [PubMed]

25. Zhu, T.; Cheng, R.; Lee, S.A.; Rajaraman, E.; Eiteman, M.A.; Querec, T.D.; Unger, E.R.; Mao, L. Continuous-flow ferrohydrodynamic sorting of particles and cells in microfluidic devices. Microfluid. Nanofluid. 2012, 13, 645-654. [CrossRef]

26. Liang, L.; Zhang, C.; Xuan, X. Enhanced separation of magnetic and diamagnetic particles in a dilute ferrofluid. Appl. Phys. Lett. 2013, 102, 234101. [CrossRef]

27. Zhang, J.; Yan, S.; Yuan, D.; Zhao, Q.; Tan, S.H.; Nguyen, N.; Li, W. A novel viscoelastic-based ferrofluid for continuous sheathless microfluidic separation of nonmagnetic microparticles. Lab Chip 2016, 16, 3947-3956. [CrossRef]

28. Chen, Q.; Li, D.; Lin, J.; Wang, M.; Xuan, X. Simultaneous separation and washing of nonmagnetic particles in an inertial ferrofluid/water coflow. Anal. Chem. 2017, 89, 6915-6920. [CrossRef]

29. Amir, S.; Besanjideh, M. Investigation of a novel microfluidic device for label-free ferrohydrodynamic cell separation on a rotating disk. IEEE Trans. Biomed. Eng. 2020, 67, 372-378.

30. Liu, Y.; Zhao, W.; Cheng, R.; Logun, M.; Zayas-Viera, M.M.; Karumbaiah, L.; Mao, L. Label-free ferrohydrodynamic separation of exosome-like nanoparticles. Lab Chip 2020, 20, 3187-3201. [CrossRef]

31. Zeng, L.; Chen, X.; Du, J.; Yu, Z.; Zhang, R.; Zhang, Y.; Yang, H. Label-free separation of nanoscale particles by an ultrahigh gradient magnetic field in a microfluidic device. Nanoscale 2021, 13, 4029-4037. [CrossRef] [PubMed]

32. Furlani, E.P. Analysis of particle transport in a magnetophoretic microsystem. J. Appl. Phys. 2006, 99, 024912. [CrossRef]

33. Hejazian, M.; Li, W.; Nguyen, N.T. Lab on a chip for continuous-flow magnetic cell separation. Lab Chip 2015, 15, 959-970. [CrossRef] [PubMed]

34. Hotta, M.; Hayashi, M.; Nagata, K. High temperature measurement of complex permittivity and permeability of $\mathrm{Fe}_{3} \mathrm{O}_{4}$ powders in the frequency range of 0.2 to $13.5 \mathrm{GHz}$. ISIJ Int. 2011, 51, 491-497. [CrossRef] 